\title{
Arsenic exposure and the seroprevalence of total hepatitis A antibodies in the US population: NHANES, 2003-2012
}

\author{
A. CARDEnAs, E. SMit, J. W. BETHEL, E. A. HOUSEMAN and M. L. Kile* \\ School of Biological and Population Health Sciences, College of Public Health and Human Sciences, Oregon \\ State University, Corvallis, OR, USA
}

Received 23 June 2015; Final revision 10 November 2015; Accepted 18 November 2015; first published online 7 January 2016

\section{SUMMARY}

We evaluated the association between urinary arsenic and the seroprevalence of total hepatitis A antibodies (total anti-HAV: IgG and IgM) in 11092 participants aged $\geqslant 6$ years using information collected in the US National Health and Nutrition Examination Survey (2003-2012). Multivariate logistic regression models evaluated associations between total anti-HAV and total urinary arsenic defined as the sum of arsenite, arsenate, monomethylarsonate and dimethylarsinate (TUA1). Effect modification by self-reported HAV immunization status was evaluated. Total anti-HAV seroprevalence was $35 \cdot 1 \%$ [95\% confidence interval (CI) 33.3-36.9]. Seropositive status was associated with higher arsenic levels and this association was modified by immunization status $(P=0 \cdot 03)$. For participants that received $\geqslant 2$ vaccine doses or did not know if they had received any doses, a positive dose-response association was observed between increasing TUA1 and odds of total anti-HAV [odds ratio (OR) 1.42, 95\% CI 1·11-1.81; and OR 1·75, 95\% CI 1.22-2.52], respectively. A positive but not statistically significant association was observed in those who received $<2$ doses (OR 1·46, 95\% CI 0.83-2.59) or no dose (OR 1.12, 95\% CI 0.98-1·30). Our analysis indicates that prevalent arsenic exposure was associated with positive total anti-HAV seroprevalence. Further studies are needed to determine if arsenic increases the risk for incident hepatitis A infection or HAV seroconversion.

Key words: Antibody responses, arsenic, hepatitis A, hepatitis immunization, immunotoxicity NHANES.

\section{INTRODUCTION}

Hepatitis A virus (HAV) is transmitted via the faecaloral route and infection causes self-limiting liver disease which is generally asymptomatic or mild but can presents with more severe symptoms including fever, jaundice and occasionally fulminant hepatitis. Globally, there are about 1.4 million cases of acute

\footnotetext{
* Author for correspondence: Dr M. L. Kile, School of Biological and Population Health Sciences, College of Public Health and Human Sciences, Oregon State University, 15 Milam, Corvallis, OR 97330.

(Email: molly.kile@oregonstate.edu)
}

hepatitis A per year with seroprevalence data suggesting that HAV affects tens of millions of people annually. In parts of the world with poor sanitation infrastructure, HAV infections tend to occur early in life with an estimated $90 \%$ of children having an infection before the age of 10 years. However, in developed countries with improved sanitation this pattern shifts and HAV infections tend to occur later in life in adolescents, adults and high-risk groups [1]. In the United States, there were 1398 reported cases of acute HAV in 2011 with the actual number of cases estimated to be between 1650 and 4370 [2]. Of these reported cases, $78 \%$ indicated they had not participated in any risk behaviours for HAV transmission such as travel to 
an endemic area, men having sex with men (MSM), injection drug use, or sex with multiple partners; nor had they been exposed to any known infected individuals [2].

In 1995, a vaccine against HAV became available in the United States which markedly reduced HAV incidence particularly after it was recommended as a routine vaccination for children in 1999. The vaccine was introduced incrementally with a 1996 recommendation from the Advisory Committee on Immunization Practices to vaccinate children living in communities with the highest disease rates followed by states and counties with elevated incidence rates [3]. Hepatitis A vaccine in children is administered in a two-dose series to children at age 12 months but it can also be provided during subsequent visits if the child has not been vaccinated at age 2 years. Adult immunization is recommended for international travellers to countries where hepatitis A is endemic, MSM, illegal drug users and other susceptible groups administered as a three-dose series along with the hepatitis B vaccine [4].

In 2009 hepatitis A immunization coverage estimates in the United States for $\geqslant 1$ and $\geqslant 2$ vaccine doses ranged from 29-58\% and 6-24\%, respectively [5]. Vaccination against HAV is very effective and studies show that protective levels of anti-HAV antibodies develop in $94-100 \%$ of adults 1 month after one dose of the vaccine and $100 \%$ have protective HAV antibody levels after two doses [6]. In children and adolescents, $97-100 \%$ have protective antibody levels 1 month after the first dose and $100 \%$ reach protective levels 1 month after the second dose [6]. Additionally, a followup study observed that after 5 years seroprotection rates were $99 \cdot 7 \%$ and $100 \%$ in 5-year-old children who had received one and two doses, respectively [7].

Following inoculation, the immune system responds by producing immunoglobulin $\mathrm{G}(\mathrm{IgG})$ antibodies to the HAV. However, immunoglobulin M (IgM) antibodies have been detected 2-3 weeks after vaccination in $8-20 \%$ of adults. In the case of HAV infection, IgM anti-HAV appear after 5-10 days before the onset of symptoms but gradually disappear with increasing IgG levels conferring long-term protection against the virus. Subsequently, epidemiologists use total anti-HAV (IgM and IgG) levels to estimate the prevalence of previous hepatitis A infection [8]. Yet despite the availability of an effective vaccine, hepatitis A remains a public health problem particularly in highly endemic regions such as Central America, South America, Africa, Middle East, South East
Asia and the South Pacific that have not fully implemented vaccination programmes $[9,10]$.

Previous studies indicate that the severity and morbidity of infectious diseases can be related to environmental pollutants and exogenous chemical exposures even at low levels [11]. The impact of environmental contaminants on the immune system has been documented in several epidemiological studies in the past decade. For example, an increased incidence of infections in children has been observed with elevated exposure to dioxins, polychlorinated biphenyls and polycyclic aromatic hydrocarbons [12, 13]. Emerging evidence also suggests that arsenic is an immunotoxicant [14]. Data from in vitro and in vivo studies show that arsenic influences macrophage differentiation and T-cell proliferation [15] and viral pathology [16]. Recent epidemiological studies report that elevated arsenic exposure during fetal development increases susceptibility to infectious diseases, including respiratory diseases, diarrhoea and overall mortality in children [17-20]. In addition, our group showed that urinary arsenic levels were inversely associated with varicella zoster virus IgG seroprevalence in the US population aged $\geqslant 6$ years [21]. Furthermore, a recent study in pregnant women reported that urinary arsenic levels was associated with increased odds of incident hepatitis E seroconversion suggesting that arsenic exposure during pregnancy may enhance susceptibility to hepatitis E viral infection [22].

While the most effective way to reduce the global burden of hepatitis A infection and transmission is through active immunization and improved sanitation, identifying environmental factors that can affect infectious diseases could inform novel prevention strategies to reduce the global burden of infectious diseases. Subsequently, the objective of this study was to investigate the cross-sectional association between arsenic exposure and immune response to HAV in a representative sample of the US population. Given that the National Health and Nutrition Examination Survey (NHANES) only measured total anti-HAV which cannot differentiate between antibodies produced by natural infection or vaccination, we limit our hypothesis to determining if there was a significant relationship between urinary arsenic levels and positive seroprevalence of total antibodies to HAV. Furthermore, we hypothesized that hepatitis A immunization would modify this association after adjusting for other risk factors because the production of antibodies (IgG) produced by vaccination be lower than levels produced after a natural infection. 


\section{METHODS}

\section{Study population}

We used data from the NHANES, a representative cross-sectional survey designed to assess the health and nutritional status of the US population, from five consecutive cycles: 2003-2012. Response rates for the five NHANES consecutive cycles were $76 \%$, $77 \cdot 36 \%, 75 \cdot 4 \%, 77 \cdot 3 \%$ and $69 \cdot 5 \%$ [23]. Serological markers for HAV were collected for participants aged $\geqslant 2$ years while urinary arsenic measurements were collected from participants aged $\geqslant 6$ years in all five survey cycles. Total hepatitis A antibodies were tested for all study participants while urinary arsenic was measured in one-third of a subsample for each survey cycle. Analytical methods for arsenic speciation and hepatitis A serology were consistent across survey cycles.

Individuals were included in this analysis if they were sampled for both urinary arsenic and total hepatitis A serology. Participants with a positive or equivocal HIV test were excluded from the analysis to avoid confounding by immunodeficiency $(n=20)$. Participants with an indeterminate serological test for HAV were also excluded from the analysis $(n=1)$. Analyses were further restricted by the availability of covariates. Total urinary arsenic was determined from urinary metabolites using two complementary approaches. Total urinary arsenic 1 (TUA1) defined as the sum of inorganic arsenic metabolites, and total urinary arsenic 2 (TUA2) defined as total urinary arsenic minus non-toxic arsenosugar metabolites described in detail in the Methods section. Depending on the exposure assessment approach, this resulted in 11092 (TUA1) and 10801 (TUA2) participants in the final analyses. Informed consent was obtained from all survey participants and study protocols were approved by the National Center for Health Statistics (NCHS) Research Ethics Review Board [24].

\section{Serological testing}

At the time of examination participants provided blood via venepuncture. The samples were processed by the Division of Viral Hepatitis, CDC using a commercial assay (Vitros, Ortho-Clinical Diagnostics, USA) and determined total antibody (IgG and IgM) to the HAV (total anti-HAV). Results are obtained as a normalized signal, relative to a lot-specific calibration cut-off value [signal/cut-off $(\mathrm{s} / \mathrm{c})]$. The total anti-HAV test relies on a photoluminescent reaction that is present for negative samples. Subsequently ratios $<1$ are considered a positive result. NHANES evaluates the anti-HAV results as antibody positive $(<0.80 \mathrm{~s} / \mathrm{c})$, borderline $(\geqslant 0.80$ and $<1.0 \mathrm{~s} / \mathrm{c})$, antibody negative $(\geqslant 1.0$ and $<4.0 \mathrm{~s} / \mathrm{c})$ or retest $(\geqslant 4.0 \mathrm{~s} / \mathrm{c})$ [25]. Thus, being positive for total anti-HAV (seropositive) indicates the person has been previously infected with HAV or has been vaccinated against HAV. Being negative for anti-HAV (seronegative) indicates the person has not been infected with HAV or has not been vaccinated against HAV. NHANES only reports results as positive, negative or intermediate as quantitative results cannot be correlated to an endpoint titre [25].

\section{Self-reported immunization}

Participants were asked to recall their hepatitis A immunization history for all NAHNES cycles and subsequently classified as receiving: $\geqslant 2$ doses, $<2$ doses, no dose, refused, and 'don't know'. Participants that refused to answer the question were excluded from the analysis $(n=7)$.

\section{Urinary arsenic assessment}

The analytical method to quantify arsenic metabolites in urine has been previously described [26] and consistent across cycles although the sensitivity of the reporting improved in 2011. Briefly, spot urine samples were obtained at the time of the physical examination in arsenic-free containers, shipped on dry ice, stored at $\leqslant-70{ }^{\circ} \mathrm{C}$ and analysed within 3 weeks of sampling at the Environmental Health Sciences Laboratory using high-performance liquid chromatography coupled to an inductively coupled-plasma dynamic reaction cell-mass spectrometry [25]. The limits of detections (LOD) for the 2003-2010 survey cycles were as follow: arsenate $(1 \cdot 0 \mu \mathrm{g} / 1)$, arsenite $(1.20 \mu \mathrm{g} / \mathrm{l})$, dimethylarsonic acid $(1.70 \mu \mathrm{g} / \mathrm{l})$, monomethylarsonic acid $(0.90 \mu \mathrm{g} / \mathrm{l})$, arsenobetaine $(0.40$ $\mu \mathrm{g} / \mathrm{l})$, arsenocholine $(0 \cdot 60 \mu \mathrm{g} / \mathrm{l})$ and TUA $(0 \cdot 74 \mu \mathrm{g} / \mathrm{l})$. The limits of detection for the 2011-2012 survey cycle were slightly different: arsenate $(0.87 \mu \mathrm{g} / \mathrm{l})$, arsenite $(0 \cdot 48 \mu \mathrm{g} / \mathrm{l})$, dimethylarsonic acid $(1 \cdot 80 \mu \mathrm{g} / \mathrm{l})$, monomethylarsonic acid $(0.89 \mu \mathrm{g} / \mathrm{l})$, arsenobetaine $(1 \cdot 19 \mu \mathrm{g} / \mathrm{l})$, arsenocholine $(0 \cdot 28 \mu \mathrm{g} / \mathrm{l})$ and TUA $(1 \cdot 25$ $\mu \mathrm{g} / \mathrm{l})$. The proportion of participants that were below the LOD for the combined survey cycles was $95.9 \%$ for arsenate, $90 \cdot 1 \%$ for arsenite, $67 \cdot 6 \%$ for monomethylarsonic acid, $18 \cdot 0 \%$ for dimethylarsonic acid, $40 \cdot 86 \%$ for arsenobetaine, $97 \cdot 60 \%$ for arsenocholine, and $1.28 \%$ for TUA. 
If a metabolite was below the LOD, a value equivalent to the LOD divided by the square root of 2 was assigned. TUA1 was defined as the sum of arsenate, arsenite, dimethylarsonic acid, and monomethylarsonic acid. Additionally, we defined TUA2 as the total urinary arsenic minus arsenobetaine and arsenocholine as a complementary approach to evaluate total arsenic exposure. For TUA2, 211 participants had urinary arsenobetaine and arsenocholine levels greater than TUA, yielding negative levels for TUA2. This would indicate that these individuals had recently consumed seafood which is naturally high in these compounds [27]. Since these organoarsenic compounds are considered to be less toxic than inorganic arsenic and its metabolites, these participants were excluded from analyses that used TUA2. In sensitivity analyses replacing these 211 values with the LOD for TUA divided by the square root of 2 did not affect the observed association (results not shown). Both approaches used to model total urinary arsenic (TUA1 and TUA2) concentration should not be influenced by organoarsenic compounds found in seafood [28].

\section{Covariates}

Previous research has described differences in urinary arsenic levels by age, sex, race and gender in the US population [28]. Additionally, the prevalence of HAV has been shown to differ by age, gender, race and country of birth [29]. Subsequently, these covariates were extracted from the five NHANES cycles and further evaluated for adjustment in multivariate models. Race/ethnicity was reported as non-Hispanic white, non-Hispanic black, Mexican American, other Hispanic and other race including multiracial. Economic status was evaluated by using the family's income to poverty ratio. Body mass index (BMI) was reported in $\mathrm{kg} / \mathrm{m}^{2}$. As suggested by the NCHS, the effect of spot urine dilution was accounted for by adjusting for creatinine $(\mathrm{mg} / \mathrm{dl})$ concentration in all multivariate models. Survey year was included in the analysis to control for temporal changes in HAV serology and arsenic exposure.

\section{Statistical analysis}

Sampling weights were calculated for the arsenic subsample of NHANES from 2003 to2012 by rescaling the survey weights of each cycle by multiplying the arsenic subsample weight by $1 / 5$, as recommended by the analytical guidelines of NHANES [30]. To account for the complex survey design implemented by NHANES, all analyses used survey weights, primary sampling units and strata. However, we also conducted a sensitivity analysis by removing the weights. Statistical significance was evaluated using a cut-off value of $\alpha \leqslant 0 \cdot 05$, and all tests performed were twotailed. Standard errors (s.E.s) and 95\% confidence intervals (CIs) were estimated using the Taylor linearization method. All statistical analyses were performed using Stata v. 12.1 (StataCorp LP, USA).

The distribution of TUA1, TUA2 and creatinine were right-skewed and subsequently natural logtransformed. The geometric mean and the s.E. for total arsenic (TUA1 and TUA2) was estimated across categories of sex, race, family income/poverty ratio, BMI, age, country of birth, and seroprevalence of total anti-HAV and evaluated using linear regression models and a Wald test adjusted for creatinine concentration to account for urine dilution. Bivariate associations between all covariates and total-anti HAV seroprevalence were evaluated and adjusted for in multivariate models if differences were observed. Multivariate logistic regression models were used to estimate the odds of being seropositive for total anti-HAV adjusting for log-transformed creatinine (continuous), age (continuous), sex, race, family income/poverty ratio (continuous), country of birth, BMI (continuous) and survey year. To evaluate effect modification the multiplicative interaction between log-transformed TUA1 and TUA2, and selfreported vaccination status were tested using a Wald test adjusting for all covariates. Odds ratios (ORs) and $95 \%$ CIs were estimated for each level of selfreported hepatitis A immunization from adjusted models that included the multiplicative interaction between exposure and immunization history to estimate the association between total arsenic exposure and hepatitis A seroprevalence.

\section{RESULTS}

The geometric means of TUA for selected population characteristics are presented in Table 1. Overall, TUA was slightly higher for females, in individuals reporting to be multiracial or of other race followed by Mexican Americans, and in individuals living in households above the poverty level for TUA2 but not for TUA1. BMI was also associated with TUA and with age. Individuals born in the United States had significantly lower TUA compared to individuals 
Table 1. Weighted geometric mean (GM) and standard error (S.E.) of total urinary arsenic levels ( $\mu$ g/l) adjusted for log-transformed creatinine, NHANES 2003-2012

\begin{tabular}{|c|c|c|c|c|}
\hline \multirow[b]{2}{*}{ Population characteristic } & \multicolumn{2}{|l|}{ TUA1* } & \multicolumn{2}{|l|}{ TUA $2 \dagger$} \\
\hline & $(N=11092)$ & GM (S.E.) & $(N=10801)$ & GM (s.E.) \\
\hline Overall & & $6 \cdot 34(1 \cdot 01)$ & & $5 \cdot 06(1 \cdot 02)$ \\
\hline Sex & & $P=0.026$ & & $P=0.074$ \\
\hline Male & 5517 & $6 \cdot 40(1 \cdot 02)$ & 5408 & $5 \cdot 19(1 \cdot 02)$ \\
\hline Female & 5575 & $6 \cdot 58(1 \cdot 01)$ & 5393 & $5 \cdot 40(1 \cdot 03)$ \\
\hline Race & & $P<0.001$ & & $P<0.001$ \\
\hline Mexican American & 2267 & $6.91(1 \cdot 02)$ & 2222 & $5.93(1.03)$ \\
\hline Other Hispanic & 821 & $8 \cdot 07(1 \cdot 02)$ & 799 & $7 \cdot 19(1 \cdot 04)$ \\
\hline Non-Hispanic white & 4593 & $6 \cdot 18(1 \cdot 02)$ & 4443 & $4.89(1.03)$ \\
\hline Non-Hispanic black & 2637 & $6 \cdot 08(1 \cdot 02)$ & 2588 & $4 \cdot 84(1 \cdot 04)$ \\
\hline Other race/multiracial & 774 & $9 \cdot 79(1 \cdot 04)$ & 749 & $10 \cdot 38(1 \cdot 06)$ \\
\hline Family income/poverty ratio & & $P=0 \cdot 2885$ & & $P=0 \cdot 010$ \\
\hline Below poverty level $\leqslant 1$ & 2771 & $6 \cdot 38(1 \cdot 02)$ & 2703 & $4.95(1.03)$ \\
\hline Above poverty level $>1$ & 8321 & $6 \cdot 50(1 \cdot 01)$ & 8098 & $5 \cdot 37(1.03)$ \\
\hline Body mass index $\left(\mathrm{kg} / \mathrm{m}^{2}\right)$ & & $P<0.001$ & & $P<0.001$ \\
\hline Underweight & 264 & $6 \cdot 44(1 \cdot 04)$ & 257 & $5 \cdot 53(1.08)$ \\
\hline Normal weight & 4342 & $6.73(1.02)$ & 4218 & $5.59(1.03)$ \\
\hline Overweight & 3132 & $6.58(1.02)$ & 3053 & $5 \cdot 38(1.03)$ \\
\hline Obese & 3354 & $6 \cdot 12(1 \cdot 01)$ & 3273 & $4.89(1.03)$ \\
\hline Age (years) & & $P<0.001$ & & $P<0 \cdot 001$ \\
\hline $6-11$ & 1420 & $7 \cdot 06(1 \cdot 02)$ & 1377 & $6 \cdot 00(1.03)$ \\
\hline $12-19$ & 2226 & $5.68(1.02)$ & 2177 & $4.00(1.03)$ \\
\hline$\geqslant 20$ & 7446 & $6 \cdot 56(1 \cdot 01)$ & 7247 & $5 \cdot 46(1 \cdot 03)$ \\
\hline Country of birth & & $P<0 \cdot 001$ & & $P<0.001$ \\
\hline United States & 8863 & $6 \cdot 16(1 \cdot 01)$ & 8619 & $4 \cdot 87(1 \cdot 03)$ \\
\hline Elsewhere & 2229 & $8.75(1.02)$ & 2182 & $8.65(1.04)$ \\
\hline Hepatitis A immunization & & $P=0 \cdot 104$ & & $P=0 \cdot 051$ \\
\hline$\geqslant 2$ doses & 2941 & $6 \cdot 68(1.02)$ & 2854 & $5.65(1.03)$ \\
\hline$<2$ doses & 273 & $6 \cdot 52(1 \cdot 03)$ & 268 & $5 \cdot 46(1 \cdot 08)$ \\
\hline 0 dose & 6603 & $6 \cdot 41(1 \cdot 01)$ & 6438 & $5 \cdot 20(1 \cdot 03)$ \\
\hline Don't know & 1275 & $6 \cdot 52(1 \cdot 02)$ & 1241 & $5 \cdot 18(1 \cdot 04)$ \\
\hline Hepatitis A (anti-HAV) & & $P<0 \cdot 001$ & & $P<0.001$ \\
\hline Seropositive & 5064 & $7 \cdot 12(1 \cdot 02)$ & 4934 & $6 \cdot 19(1 \cdot 03)$ \\
\hline Seronegative & 6028 & $6 \cdot 17(1 \cdot 01)$ & 5867 & $4 \cdot 88(1.03)$ \\
\hline
\end{tabular}

* TUA1 $=\mathrm{As}^{\mathrm{III}}+\mathrm{As}^{\mathrm{V}}+\mathrm{MMA}+\mathrm{DMA}$.

$\dagger$ TUA2 = total urinary As - arsenocholine - arsenobetaine.

$\$$ Models adjusted for log-transformed creatinine.

born elsewhere. Urinary arsenic was higher in seropositive anti-HAV participants compared to seronegative individuals. No differences in TUA were observed by self-reported immunization status to HAV.

The total anti-HAV seroprevalence (e.g. seropositive) for selected population characteristics are presented in Table 2. Across all 10 years, the overall seropositive prevalence was estimated to be $35 \cdot 1 \%$ (95\% CI 33-3-36.9). Seropositive prevalence of total anti-HAV was highest in Mexican Americans (75\%), participants living below the poverty level (45\%), children aged 6-11 years (44\%), participants in the lowest urinary creatinine tertile $(38 \%)$, individuals not born in the United States $(77 \%)$ and in individuals that selfreported receiving $\geqslant 2$ doses of hepatitis A vaccine $(52 \%)$. Total anti-HAV seropositive prevalence was elevated for participants in the highest quartile of arsenic exposure compared to the third, second and first quartiles for TUA1 $(43 \%)$ or TUA2 (42\%). In our restricted sample for TUA1, a total of $26 \cdot 51 \%$ individuals reported receiving $\geqslant 2$ doses of hepatitis A vaccine, $2 \cdot 46 \%$ reported receiving $<2$ doses, $59.53 \%$ participants reported receiving no dose and $11.49 \%$ participants reported not knowing whether 
Table 2. Hepatitis A seroprevalence (total anti-HAV) by demographic characteristics: NHANES: 2003-2012

\begin{tabular}{|c|c|c|c|c|}
\hline \multirow[b]{3}{*}{ Population characteristics } & \multicolumn{4}{|c|}{ Hepatitis A seroprevalence (total anti-HAV) } \\
\hline & \multicolumn{2}{|l|}{ TUA1* } & \multicolumn{2}{|l|}{ TUA $2 \dagger$} \\
\hline & $(N=11092)$ & Seropositive $(95 \% \mathrm{CI})$ & $(N=10801)$ & Seropositive $(95 \% \mathrm{CI})$ \\
\hline Overall prevalence & 5064 & $35 \cdot 1 \%(33 \cdot 3-36 \cdot 9)$ & 4934 & $34 \cdot 9 \%(33 \cdot 1-36 \cdot 7)$ \\
\hline Sex & & $P=0.926$ & & $P=0.936$ \\
\hline Male & 5517 & $35 \cdot 0 \%(32 \cdot 7-37 \cdot 4)$ & 5408 & $34 \cdot 9(32 \cdot 5-37 \cdot 3)$ \\
\hline Female & 5575 & $35 \cdot 1 \%(33 \cdot 1-37 \cdot 1)$ & 5393 & $34 \cdot 9(32 \cdot 9-36 \cdot 9)$ \\
\hline Race & & $P<0.001$ & & $P<0.001$ \\
\hline Mexican American & 2267 & $75 \cdot 5 \%(72 \cdot 9-78 \cdot 1)$ & 2222 & $75 \cdot 4 \%(72 \cdot 8-78 \cdot 1)$ \\
\hline Other Hispanic & 821 & $63 \cdot 2 \%(58 \cdot 5-68 \cdot 0)$ & 799 & $63 \cdot 1 \%(58 \cdot 4-67 \cdot 8)$ \\
\hline Non-Hispanic white & 4593 & $25 \cdot 5 \%(23 \cdot 9-27 \cdot 2)$ & 4443 & $25 \cdot 2 \%(23 \cdot 5-26 \cdot 9)$ \\
\hline Non-Hispanic black & 2637 & $36 \cdot 9 \%(34 \cdot 3-39 \cdot 6)$ & 2588 & $36 \cdot 9 \%(34 \cdot 4-39 \cdot 6)$ \\
\hline Other race/multiracial & 774 & $57 \cdot 8 \%(52 \cdot 5-62 \cdot 9)$ & 749 & $57 \cdot 8 \%(52 \cdot 6-62 \cdot 9)$ \\
\hline Family income/poverty ratio & & $P<0.001$ & & $P<0 \cdot 001$ \\
\hline Below poverty level $\leqslant 1$ & 2771 & $45 \cdot 4 \%(31 \cdot 9-34 \cdot 9)$ & 2703 & $45 \cdot 3 \%(41 \cdot 8-48 \cdot 9)$ \\
\hline Above poverty level >1 & 8321 & $33 \cdot 1 \%(31 \cdot 4-34 \cdot 9)$ & 8098 & $32 \cdot 9 \%(31 \cdot 2-34 \cdot 7)$ \\
\hline Body mass index $\left(\mathrm{kg} / \mathrm{m}^{2}\right)$ & & $P=0.138$ & & $P=0.168$ \\
\hline Underweight & 264 & $31 \cdot 6 \%(24 \cdot 2-39 \cdot 1)$ & 257 & $32 \cdot 6 \%(24 \cdot 9-40 \cdot 3)$ \\
\hline Normal weight & 4342 & $35 \cdot 8 \%(33 \cdot 2-38 \cdot 4)$ & 4218 & $35 \cdot 8 \%(33 \cdot 2-38 \cdot 5)$ \\
\hline Overweight & 3132 & $35 \cdot 9 \%(33 \cdot 6-38 \cdot 2)$ & 3053 & $35 \cdot 6 \%(33 \cdot 4-37 \cdot 8)$ \\
\hline Obese & 3354 & $33 \cdot 6 \%(31 \cdot 3-35 \cdot 9)$ & 3273 & $33 \cdot 3 \%(30 \cdot 9-35 \cdot 7)$ \\
\hline Age (years) & & $P<0.001$ & & $P<0.001$ \\
\hline $6-11$ & 1420 & $44 \cdot 2 \%(39 \cdot 7-49 \cdot 3)$ & 1377 & $44 \cdot 8 \%(39 \cdot 6-49 \cdot 9)$ \\
\hline $12-19$ & 2226 & $36 \cdot 4 \%(32 \cdot 3-40 \cdot 4)$ & 2177 & $35 \cdot 9 \%(31 \cdot 6-40 \cdot 3)$ \\
\hline$\geqslant 20$ & 7446 & $33 \cdot 9 \%(33 \cdot 4-37 \cdot 4)$ & 7247 & $33 \cdot 8 \%(32 \cdot 0-35 \cdot 6)$ \\
\hline Creatinine (mg/dl) (tertiles) & & $P<0.001$ & & $P<0.001$ \\
\hline$(6-75)$ & 3367 & $37 \cdot 9 \%(35 \cdot 5-40 \cdot 3)$ & 3175 & $37 \cdot 8 \%(35 \cdot 3-40 \cdot 2)$ \\
\hline$(76-145)$ & 3850 & $36 \cdot 7 \%(34 \cdot 2-39 \cdot 1)$ & 3804 & $36 \cdot 5 \%(34 \cdot 1-38 \cdot 9)$ \\
\hline$(146-800)$ & 3875 & $30 \cdot 7 \%(28 \cdot 3-33 \cdot 1)$ & 3822 & $30 \cdot 6 \%(28 \cdot 2-33 \cdot 0)$ \\
\hline Country of birth & & $P<0.001$ & & $P<0.001$ \\
\hline United States & 8863 & $27 \cdot 9 \%(26 \cdot 3-29 \cdot 6)$ & 8619 & $27 \cdot 7 \%(26 \cdot 1-29 \cdot 4)$ \\
\hline Elsewhere & 2229 & $76 \cdot 9 \%(73 \cdot 7-80 \cdot 0)$ & 2182 & $76 \cdot 6 \%(73 \cdot 3-79 \cdot 9)$ \\
\hline TUA1 ( $\mu \mathrm{g} / 1)$ (quartiles) & & $P<0.001$ & & \\
\hline $2 \cdot 69-4 \cdot 14$ & 2470 & $32 \cdot 9 \%(30 \cdot 2-35 \cdot 6)$ & - & - \\
\hline $4 \cdot 15-5 \cdot 89$ & 2755 & $32 \cdot 4 \%(29 \cdot 3-34 \cdot 8)$ & - & - \\
\hline $5 \cdot 90-8 \cdot 54$ & 2848 & $31 \cdot 9 \%(29 \cdot 4-34 \cdot 5)$ & - & - \\
\hline $8 \cdot 55-628$ & 3019 & $43 \cdot 1 \%(40 \cdot 1-46 \cdot 0)$ & - & - \\
\hline TUA2 ( $\mu \mathrm{g} / \mathrm{l})$ (quartiles) & & & & $P<0.001$ \\
\hline $0-2 \cdot 72$ & - & - & 2460 & $31 \cdot 6 \%(29 \cdot 1-34 \cdot 1)$ \\
\hline $2 \cdot 73-5 \cdot 3$ & - & - & 2661 & $33 \cdot 6 \%(30 \cdot 9-36 \cdot 3)$ \\
\hline $5 \cdot 31-9 \cdot 88$ & - & - & 2824 & $32 \cdot 9 \%(29 \cdot 7-36 \cdot 0)$ \\
\hline $9 \cdot 89-718 \cdot 9$ & - & - & 2856 & $41 \cdot 8 \%(38 \cdot 9-44 \cdot 8)$ \\
\hline Hepatitis A immunization & & $P<0.001$ & & $P<0.001$ \\
\hline$\geqslant 2$ doses & 2941 & $51 \cdot 9 \%(48 \cdot 8-55 \cdot 0)$ & 2854 & $52 \cdot 0 \%(48 \cdot 9-55 \cdot 2)$ \\
\hline$<2$ doses & 273 & $41 \cdot 6 \%(33 \cdot 3-49 \cdot 8)$ & 268 & $41 \cdot 9 \%(33 \cdot 6-50 \cdot 4)$ \\
\hline 0 dose & 6603 & $29 \cdot 1 \%(27 \cdot 3-30 \cdot 9)$ & 6438 & $28 \cdot 8 \%(26 \cdot 9-30 \cdot 6)$ \\
\hline Don’t know & 1275 & $35 \cdot 0 \%(31 \cdot 1-38 \cdot 9)$ & 1241 & $35 \cdot 3 \%(31 \cdot 5-39 \cdot 1)$ \\
\hline
\end{tabular}

CI, Confidence interval.

$* \mathrm{TUA} 1=\mathrm{As}^{\mathrm{III}}+\mathrm{As}^{\mathrm{V}}+\mathrm{MMA}+\mathrm{DMA}$

$\dagger$ TUA2 $=$ total urinary As - arsenocholine - arsenobetaine.

they received a dose of the HAV vaccine. A similar self-reported HAV immunization distribution was observed for TUA2.
The ORs for the association between TUA and total anti-HAV seropositive prevalence were estimated for each self-reported category of hepatitis A 
Table 3. Adjusted odds ratios* for the association of total urinary arsenic levels and total anti-HAV seropositive prevalence by self-reported immunization status

\begin{tabular}{lllll}
\hline \hline Exposure assessment & $\begin{array}{l}\geqslant 2 \text { doses } \\
\text { aOR }(95 \% \mathrm{CI})\end{array}$ & $\begin{array}{l}<2 \text { doses } \\
\mathrm{aOR}(95 \% \mathrm{CI})\end{array}$ & $\begin{array}{l}0 \text { dose } \\
\mathrm{aOR}(95 \% \mathrm{CI})\end{array}$ & $\begin{array}{l}\text { Don't know } \\
\mathrm{aOR}(95 \% \mathrm{CI})\end{array}$ \\
\hline Ln TUA1 $\dagger$ & $1 \cdot 42(1 \cdot 11-1 \cdot 81)$ & $1 \cdot 46(0 \cdot 83-2 \cdot 59)$ & $1 \cdot 12(0 \cdot 98-1 \cdot 30)$ & $1 \cdot 75(1 \cdot 22-2 \cdot 52)$ \\
Ln TUA2 & $1 \cdot 17(1 \cdot 04-1 \cdot 31)$ & $1 \cdot 26(0 \cdot 95-1 \cdot 67)$ & $1 \cdot 07(0 \cdot 99-1 \cdot 16)$ & $1 \cdot 20(0 \cdot 97-1 \cdot 48)$ \\
\hline \hline
\end{tabular}

aOR, Adjusted odds ratio; CI, confidence interval.

* Odds ratios from a logistic regression model that included the interaction between arsenic exposure (TUA1 or TUA2) and hepatitis A vaccine adjusted for log-transformed creatinine, age, sex, race, family income/poverty ratio, country of birth, body mass index and survey year.

$\dagger \mathrm{TUA} 1=\mathrm{As}^{\mathrm{III}}+\mathrm{As}^{\mathrm{V}}+\mathrm{MMA}+\mathrm{DMA}$.

$\ddagger \mathrm{TUA} 2$ = total urinary As - arsenocholine - arsenobetaine (excluding negatives levels due to arsenocholine + arsenobetaine $>$ total As).

immunization from adjusted logistic regression models that included the multiplicative interaction between log-transformed urinary arsenic (TUA1 and TUA2) and self-reported hepatitis A immunization (Table 3). In multivariate models the multiplicative interaction between continuous log-transformed TUA1 and self-reported hepatitis A vaccination as categorical outcome ( $\geqslant 2$ doses, $<2$ doses, no dose, 'don't know') was statistically significant (Wald $P=$ 0.032 ) as well as with TUA2 (Wald $P=0.010$ ) after adjusting for log-transformed creatinine, age, sex, race, BMI, family income/poverty ratio, country of birth and survey year. In adjusted analysis, participants that self-reported receiving the complete two doses of hepatitis A vaccine had $42 \%$ greater odds of testing positive for total anti-HAV for every unit increase in log-transformed TUA1 levels (OR 1.42, $95 \%$ CI $1 \cdot 11-1 \cdot 81)$. Similarly, the adjusted odds of testing positive for total anti-HAV (e.g. seropositive) increased by $17 \%$ for every unit increase in logtransformed TUA2 (OR 1.17, 95\% CI 1.04-1.31). The odds of testing positive for total anti-HAV increased by $75 \%$ for every unit increase in logtransformed TUA1 levels in participants reporting not knowing whether they have received a hepatitis A vaccine dose (OR 1·75, 95\% CI 1.22-2.52). A marginal increase of $20 \%$ was also observed for this group in the adjusted odds of being positive for total anti-HAV for every unit increase in log-transformed TUA2 (OR 1·20, 95\% CI 0.97-1·48). No association was observed for participants that self-reported receiving $<2$ doses of hepatitis A vaccine for TUA1 (OR $1 \cdot 46,95 \%$ CI $0 \cdot 83-2 \cdot 59$ ) or TUA2 (OR $1 \cdot 26,95 \%$ CI $0.95-1.67)$ or for individuals that reported receiving no dose of the vaccine for TUA1 (OR $1 \cdot 12,95 \%$ CI $0 \cdot 98-1 \cdot 30$ ) or TUA2 (OR $1 \cdot 07,95 \%$
CI 0.99-1·16). Results were consistent when models were stratified by self-reported vaccination status or analysed without the sampling design and weights (results not shown). The observed relationship remained consistent when total arsenic was modelled by quartiles of exposure of TUA1 (Fig. 1) or TUA2 (Fig. 2). Age did not modify the observed association.

\section{DISCUSSION}

This cross-sectional study observed that higher arsenic exposure levels were associated with a greater probability of total anti-HAV seropositive prevalence in the US population aged $\geqslant 6$ years.

This dose-dependent association was consistently positive in all self-reported immunization strata but only reaches statistical significance in individuals that reported receiving $\geqslant 2$ doses of HAV immunization and for participants that did not know whether they had received HAV immunization. Given the cross-sectional nature of this study and reliance on total anti-HAV as the outcome, we cannot ascertain whether arsenic exposure is related to increased risk of hepatitis A infection or if toxic exposure modulates the immunological response to vaccination since a positive total anti-HAV test is not able to distinguish between a present, previous infection, or vaccineinduced immunity [31]. This is because hepatitis A IgM indicates a current underlying infection but hepatitis A IgG indicates immunity or recovery from a previous natural infection [32]. Furthermore, it is unknown if anti-HAV levels diminish over time. However, total anti-HAV is commonly used in epidemiological studies to measure the overall prevalence of previous infections [8]. The active production of the IgG antibody by vaccination can be 10 - to 100 -fold 

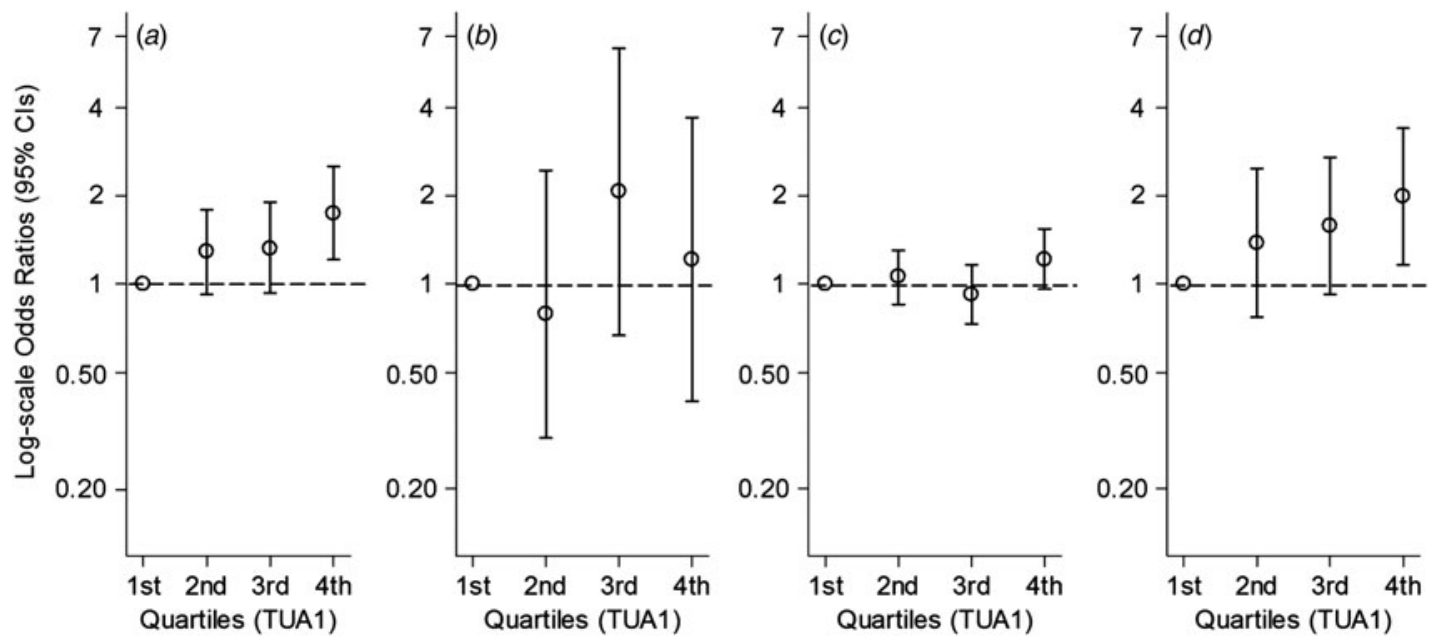

Fig. 1. Adjusted odds ratios* for the association between total urinary arsenic (TUA1) in quartiles and total anti-HAV seropositive prevalence by self-reported HAV immunization: $(a)$ received $\geqslant 2$ doses, $(b)$ received $<2$ doses, $(c) 0$ dose and (d) 'don't know'. [* Odds ratios from a logistic regression model that included the interaction between arsenic exposure by quartiles (TUA1) and hepatitis A immunization adjusted for log-transformed creatinine, age, sex, race, family income/ poverty ratio, country of birth, body mass index and survey year.]
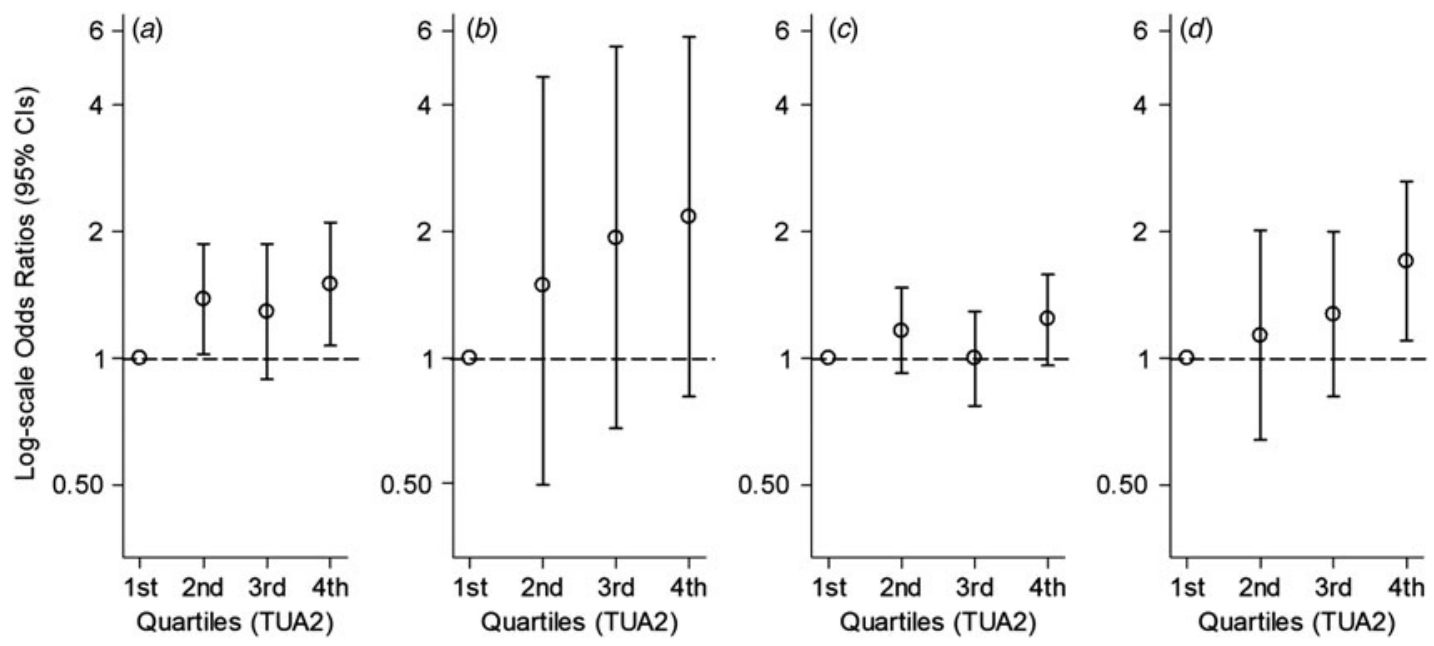

Fig. 2. Adjusted odds ratios* for the association between total urinary arsenic (TUA2) in quartiles and total anti-HAV seropositive prevalence by self-reported HAV immunization: $(a)$ received $\geqslant 2$ doses, $(b)$ received $<2$ doses, $(c) 0$ dose and (d) 'don't know'. [* Odds ratios from a logistic regression model that included the interaction between arsenic exposure by quartiles (TUA2) and hepatitis A immunization adjusted for log-transformed creatinine, age, sex, race, family income/ poverty ratio, country of birth, body mass index and survey year.]

lower compared to IgG antibody levels produced by natural infections and in many instances present below the detection limit for many commercial assays including the assay used by NHANES [8]. This is also highlighted in our data that shows that only $52 \%$ of individuals that self-reported receiving $\geqslant 2$ doses of HAV vaccine tested seropositive for total anti-HAV. It is possible, however, that this could also originate from recall bias from participants as the sensitivity of self-reported HAV immunization has been estimated to be $63 \%$ compared to electronic medical records [33]. While we cannot rule out misclassification it is likely to be non-differential in relationship to urinary arsenic concentrations which is unlikely to be known by an individual. Thus, a possible explanation of our findings is that arsenic exposure may be associated with a lower protective immunity provided by the HAV immunization, which may subsequently result in HAV infection yielding a stronger antibody response. 
This interpretation contradicted our initial hypothesis that HAV vaccination would be protective of the immunotoxic effect from arsenic exposure. Additionally, the absence of an association for individuals receiving no dose of the vaccine or $<2$ doses could be attributed to the lack of protection of not receiving the adequate immunization so differential exposure to arsenic, either high or low, would not affect the susceptibility to infection or immune response of these subgroups. The observed significant association for protected individuals (self-reported receiving two doses) or the mixture of protected/unprotected individuals (self-reporting not knowing whether they received any dose) could be influenced by arsenic exposure levels by either: $(a)$ decreasing protecting immunity of the vaccine or $(b)$ altering antibody response to the hepatitis A vaccine. Arsenic could contribute to the dysregulation of IgG or IgM antibody production in vaccinated participants yielding higher detectable levels of total anti-HAV. Additional studies are needed to test the hypothesis that arsenic reduces vaccine-induced immunity conferred by HAV vaccination or whether it actually increases susceptibility to hepatitis A infection. A recent epidemiological investigation demonstrated that elevated urinary arsenic levels during pregnancy increase the incidence of hepatitis E virus seroconversion, suggesting that environmental arsenic exposure enhances susceptibility to hepatitis E viral infection [22]. Further studies are therefore warranted to determine if arsenic exposure can influence susceptibility to hepatitis A.

There is considerable evidence that arsenic affects the immune system. Experimental studies in zebrafish have demonstrated that exposure of arsenic at environmentally relevant concentrations compromised the overall innate and adaptive immune system [34, 35]. In vivo studies have also shown that arsenic exposure increases the percentage and total levels of CD8+ T cells, and decreases cytokine production [36]. Alterations in gene expression related to the immune response, including genes involved in T-cell receptor signalling have also been observed with increased exposure to inorganic arsenic $[37,38]$. $\mathrm{T}$ cells play a key role in the immune response to infections and vaccine-induced immunity that could modulate arsenic's induced immunotoxicity [39]. Recent evidence from birth cohorts have demonstrated that in utero exposure to arsenic altered the $\mathrm{CD} 4+/ \mathrm{CD} 8+$ $\mathrm{T}$-cell ratios and also slightly decreased the proportion of B cells in newborns [40, 41]. The key role of T cells in stimulating $\mathrm{B}$ cells for the production of antibodies makes T-cell regulation a likely target for the observed association.

This study has several strengths including the use of a large recently collected representative sample of the US population across a time period of 9 years. Arsenic exposure levels represent exposure at environmentally relevant concentrations and our complementary approach of using two different methods for assessing exposure yielded consistent results. The use of several biomarkers of arsenic exposure and the meticulous quality control procedures for the analysis and collection of specimens implemented by NHANES are also important strengths. However, our study also has several limitations. Primarily, total anti-HAV seropositivity is unable to differentiate between a present or previous infection and vaccine-induced immunity. Furthermore, the assay used by NHANES is qualitative and cannot be correlated to a titre endpoint. The data were collected cross-sectionally and therefore we cannot evaluate temporality of this association between arsenic and anti-HAV response. While we were able to adjust for major risk factors of HAV infection identified in the literature, the possibility of residual confounding cannot be ruled out. Moreover, despite the strengths of using a biomarker of internal dose to ascertain arsenic exposure, urinary arsenic was measured only at one point in time and we acknowledge that exposure may fluctuate over time. The possibility for reverse causation of viral hepatitis modifying the metabolism and excretion of urinary arsenic cannot be ruled out and should be considered in future studies. NHANES does not sample institutionalized or homeless individuals and it is expected that the prevalence of HAV would be much higher in these individuals.

\section{CONCLUSION}

Overall, elevated arsenic exposure was positively associated with the seroprevalence of total anti-HAV in participants receiving $\geqslant 2$ doses of HAV vaccine and for individuals unable to recall their immunization history after adjusting for major risk factors and potential confounders. The elevated prevalence of serological markers suggests that arsenic exposure may alter the immune response to HAV and this relationship was modified by self-reported immunization status. This study generates hypotheses for further study that are designed to understand whether arsenic exposure alters the immune response to hepatitis A or alters the response to vaccine-induced immunity. 


\section{ACKNOWLEDGEMENTS}

This work was supported by US NIH (NIEHS) grants K01 ES017800, P30 ES000210 and R01 ES023441.

\section{DECLARATION OF INTEREST}

None.

\section{REFERENCES}

1. WHO. Hepatitis A. Fact sheet no. 328. World Health Organization, 2014.

2. CDC. Surveillance for viral hepatitis - United States, 2011. Centers for Disease Control and Prevention, 2013.

3. Wasley A, Fiore A, Bell BP. Hepatitis A in the era of vaccination. Epidemiologic Reviews 2006; 28: 101-111.

4. Committee on Infectious Diseases. Hepatitis A vaccine recommendations. Pediatrics 2007; 120: 189-199.

5. CDC. Hepatitis A vaccination coverage among US children aged 12-23 months-immunization information system sentinel sites, 2006-2009. Morbidity and Mortality Weekly Report 2010; 59: 776.

6. Ashur Y, et al. Comparison of immunogenicity of two hepatitis A vaccines - VAQTA ${ }^{\circledR}$ and HAVRIX $^{\circledR}$ - in young adults. Vaccine 1999; 17: 2290-2296.

7. Espul C, et al. Five-year follow-up of immune response after one or two doses of inactivated hepatitis A vaccine given at 1 year of age in the Mendoza Province of Argentina. Journal of Viral Hepatitis 2015; 22: 453-458.

8. Fiore AE, Wasley A, Bell BP. Prevention of hepatitis A through active or passive immunization. Recommendations of the Advisory Committee on Immunization Practices (ACIP). Morbidity and Mortality Weekly Report 2006; 55: 1-23.

9. CDC. Hepatitis A information for health professionals. Centers for Disease Control and Prevention, 2013.

10. WHO. Global policy report on the prevention and control of viral hepatitis in WHO Member States. World Health Organization, 2013.

11. Colosio C, et al. Low level exposure to chemicals and immune system. Toxicology and Applied Pharmacology 2005; 207: 320-328.

12. Weisglas-Kuperus $\mathbf{N}$, et al. Immunologic effects of background prenatal and postnatal exposure to dioxins and polychlorinated biphenyls in Dutch infants. Pediatric Research 1995; 38: 404-410.

13. Jedrychowski W, et al. Prenatal ambient air exposure to polycyclic aromatic hydrocarbons and the occurrence of respiratory symptoms over the first year of life. European Journal of Epidemiology 2005; 20: 775-782.

14. Dangleben NL, Skibola CF, Smith MT. Arsenic immunotoxicity: a review. Environmental Health 2013; 12: 73.

15. Lemarie A, et al. Human macrophages constitute targets for immunotoxic inorganic arsenic. Journal of Immunology 2006; 177: 3019-3027.

16. Dangleben NL, Skibola CF, Smith MT. Arsenic immunotoxicity: a review. Environmental Health 2013; 12: 73.
17. Farzan SF, et al. In utero arsenic exposure and infant infection in a United States cohort: A prospective study. Environmental Research 2013; 126: 24-30.

18. Parvez F, et al. A prospective study of respiratory symptoms associated with chronic arsenic exposure in Bangladesh: findings from the Health Effects of Arsenic Longitudinal Study (HEALS). Thorax 2010; 65: 528-533.

19. Rahman A, et al. Arsenic exposure in pregnancy increases the risk of lower respiratory tract infection and diarrhea during infancy in Bangladesh. Environmental Health Perspectives 2011; 119: 719.

20. Rahman M, et al. Increased childhood mortality and arsenic in drinking water in Matlab, Bangladesh: a population-based cohort study. PLOS ONE 2013; 8: e55014.

21. Cardenas A, et al. Arsenic exposure and prevalence of the varicella zoster virus in the United States: NHANES (2003-2004 and 2009-2010). Environmental Health Perspectives 2015; 123: 590-596.

22. Heaney CD, et al. Arsenic exposure and hepatitis E virus infection during pregnancy. Environmental Research 2015; 142: 273-280.

23. CDC. NHANES response rates and population totals. Centers for Disease Control and Prevention, 2013.

24. CDC. National Center for Health Statistics. NCHS Research Ethics Review Board (ERB) Approval. Centers for Disease Control and Prevention, 2012.

25. CDC. Laboratory Procedure Manual: Hepatitis A Antibody: HAV T-Anti-HAV Total VITROS Immunodiagnostic Products (ref. 6801823). Centers for Disease Control and Prevention, 2014.

26. Caldwell KL, et al. Levels of urinary total and speciated arsenic in the US population: National Health and Nutrition Examination Survey 2003-2004. Journal of Exposure Science and Environmental Epidemiology 2009; 19: 59-68.

27. Mürer AJ, et al. Effect of seafood consumption on the urinary level of total hydride-generating arsenic compounds. Instability of arsenobetaine and arsenocholine. Analyst 1992; 117: 677-680.

28. Caldwell KL, et al. Levels of urinary total and speciated arsenic in the US population: National Health and Nutrition Examination Survey 2003-2004. Journal of Exposure Science and Environmental Epidemiology 2008; 19: 59-68.

29. Wasley A, Samandari T, Bell BP. Incidence of hepatitis A in the United States in the era of vaccination. Journal of the American Medical Association 2005; 294: 194 201.

30. CDC. National Center for Health Statistics: National Health and Nutrition Examination Survey: Analytic Guidelines, 1999-2010. Centers for Disease Control and Prevention, 2013.

31. Lemon SM. Type A viral hepatitis: epidemiology, diagnosis, and prevention. Clinical Chemistry 1997; 43: 1494-1499.

32. Brundage SC, Fitzpatrick AN. Hepatitis A. American Family Physician 2006; 73: 2162-2168. 
33. Rolnick S, et al. Self-report compared to electronic medical record across eight adult vaccines: do results vary by demographic factors? Vaccine 2013; 31: 3928-3935.

34. Nayak AS, Lage CR, Kim CH. Effects of low concentrations of arsenic on the innate immune system of the zebrafish (Danio rerio). Toxicological Sciences 2007; 98: 118-124.

35. Mattingly CJ, et al. Perturbation of defense pathways by low-dose arsenic exposure in zebrafish embryos. Environmental health perspectives 2009; 117: 981.

36. Kozul CD, et al. Low-dose arsenic compromises the immune response to influenza A infection in vivo. Environmental Health Perspectives 2009; 117: 1441.

37. Andrew AS, et al. Drinking-water arsenic exposure modulates gene expression in human lymphocytes from a US population. Environmental Health Perspectives 2008; 116: 524.

38. Biswas R, et al. Analysis of T-cell proliferation and cytokine secretion in the individuals exposed to arsenic. Human \& Experimental Toxicology 2008; 27: 381-386.

39. Belkaid Y, Rouse BT. Natural regulatory $T$ cells in infectious disease. Nature Immunology 2005; 6: 353-360.

40. Kile ML, et al. Effect of prenatal arsenic exposure on DNA methylation and leukocyte subpopulations in cord blood. Epigenetics 2014; 9: 774-782.

41. Koestler DC, et al. Differential DNA methylation in umbilical cord blood of infants exposed to low levels of arsenic in utero. Environmental Health Perspectives 2013; 121: 971-977. 